\title{
Comparison of clinical, radionuclide, and radiographic features of osteoarthritis of the hands
}

\author{
D G Macfarlane, J C Buckland-Wright, P Emery, I Fogelman, B Clark, J Lynch
}

\begin{abstract}
Simultaneous clinical, scintigraphic, and macroradiographic assessments were carried out on 32 patients with hand osteoarthritis and the results at entry and one year reported. The presence and growth of osteophyte correlated with symptoms and a positive scan. The scan did not detect the radiographic features of juxta-articular radiolucencies, subchondral sclerosis, or cartilage thinning. Osteophytes, particularty when fast growing, produce pain, a 'hot' scan, and may predict disintegration of joint architecture.
\end{abstract}

Conventional radiography is used to identify the features of osteoarthritis of the hands, but the disease is well established at this stage and it is not known which features represent disease and which repair. ${ }^{1}$ Long term studies of progression are hampered by difficulty in detecting early cases, which may be asymptomatic, the slow disease process, and the poor resolution of $x$ ray features. Isotope bone scanning is a sensitive technique and can detect early changes in bone, blood flow and metabolism and in osteoarthritis gives a distinctive, localised, well defined hot spot in the typical distribution of thumb base and distal interphalangeal joints, but also in the wrist and metacarpophalangeal joints. ${ }^{23} \mathrm{~A}$ study comparing scintigraphy with conventional radiography suggested that the scan may detect early or active disease, which may either resolve or progress to irreversible damage. ${ }^{4}$ The study was limited because the highly sensitive but non-specific scan image was compared with a poor resolution standard anteroposterior hand radiograph in which the anatomical changes could only be graded on a four point severity scale.

The technique of microfocal radiography uses a small source of $x$ rays $(6-8 \mu \mathrm{m})$ and produces high magnification, high resolution macroradiographs, ${ }^{56}$ from which accurate measurements of individual $x$ ray features can be made at each joint in the hand. ${ }^{7}$ It also permits the detection of change over a reasonably short time span. ${ }^{8}$ We compared measurements from bone scan imaging, clinical observation, and microfocal radiography and applied statistical analysis in an effort to define the pathological processes taking place in osteoarthritis.

\section{Patients and methods}

Thirty two patients (three male, 29 female, mean age at first visit 62 (SD 10) years, mean duration of disease 11.6 (SD 10) years) com- pleted the study. All had evidence of two of the three following features in the hands on conventional radiography: subchondral sclerosis, joint space narrowing, and osteophytes. Sixteen had interphalangeal nodes at entry, defined as one visible swelling on one joint plus three or more palpable or visible nodes on other hand joints. With these criteria 16 patients were non-nodal at entry, but nine of them fulfilled these criteria for nodality at the end of the study. Thus at 18 months 25/32 had nodal osteoarthritis of the hands. All were carefully examined to exclude other types of arthritis and all were seronegative for rheumatoid factor and had a normal erythrocyte sedimentation rate. The patients were allowed to continue taking drugs as required throughout the study. Macroradiographs of the hands of 10 non-arthritic subjects (five male, five female) were included to determine the normal morphology of the $x$ ray features to be measured. The mean age of this group was $41 \cdot 1$ years.

\section{CLINICAL ASSESSMENT}

Patients were examined at six monthly intervals by a single observer for 18 months, making four visits in all. Clinical assessment and microfocal radiography were carried out at each visit and on the same day, and bone scanning was added at the first and third visits. Assessment was made of tenderness over the distal interphalangeal, proximal interphalangeal, metacarpophalangeal, and carpometacarpal joints of the thumb and the radial and ulnar portions of the wrist by direct pressure and graded on a four point severity scale: $0=$ no pain; $1=$ tender, $2=$ tender and wince, $3=$ wince and withdraw. ${ }^{9}$ Heberden's and Bouchard's nodes were assessed by observation and palpation on the radial and ulnar sides of each finger and scored for number and for size on a five point scale: $0=a b s e n t$, 1 =palpable, 2 =small, $3=$ medium, 4=large.

\section{MICROFOCAL RADIOGRAPHY}

Stereopair macroradiographs were prepared of the right and left hands and wrists of each patient, excluding the terminal interphalangeal joint of the thumb, which would have required an additional $x$ ray plate. The hand was placed in a stereotactic unit, positioned close to the source and displaced by $6 \mathrm{~mm}$ between the first and second $x$ ray exposure. ${ }^{56}$ The stereopair macroradiographs thus obtained were examined under a large format stereoscope (Ross Instruments, Salisbury, UK) for three dimensional evaluation of the joint structures. The right 
sided, back illuminated image of the stereoscope contained a digitiser tablet linked to a MOP-videoplan (Carl Zeiss, Hertfordshire, UK). A cross wire cursor was used to outline the structure in the macroradiograph overlying the digitiser. ${ }^{5610}$ The data initially recorded on microcomputer disks were transferred onto one large disk on an IBM PC/AT using a standard format for each patient/hand/visit and recorded so that comparisons could be made of data both within and between patients.

The following $x$ ray features were measured: (1) Joint space width: the interbone distance representing the space occupied by opposing articular cartilage. Where this was reduced, measurements were made across the narrowest part of the joint if it was uneven and across the midline when it was even.

(2) Subchondral sclerosis: the thickness in millimetres of the subchondral cortex was measured at three equidistant points along the margin of each joint and the readings averaged. (3) Osteophytes: the number and area in square millimetres were measured at the articular margins and capsular insertions on the radial and ulnar sides of the proximal and distal ends of each joint.

(4) Juxta-articular radiolucencies: these radiological features of osteoarthritis have been referred to by Altman et al as erosions. ${ }^{11}$ The number and area in square millimetres were measured using a program developed for the evaluation of erosions in rheumatoid arthritis. ${ }^{12}$

\section{BONE SCAN}

A four hour scintigraphic image was obtained of the hands after injection of $555 \mathrm{MBq}$ of technetium-99m methylene diphosphonate by acquiring counts for 15 minutes with a gammacamera. The films were read at one sitting and were compared rather than read blind. The intensity of the image from each joint was assessed by one observer and scored: $1=$ suspicious, $2=$ positive, $3=$ strongly positive, and $4=$ intense. The wrist was considered as two units: radial and ulnar.

\section{ANALYSIS}

For each joint in the hand and the two wrist units the bone scan, $x$ ray features, and clinical features were collated and the distribution of each throughout the hand was determined. Correlations between features were calculated using Kendall's tau $\mathrm{C}$, and changes between visits were tested for significance using Wilcoxon's signed ranks test for imaging data and Wilcoxon's signed ranks test for clinical data. All statistical evaluations were carried out on an IBM PC/AT using SPSS/PC software, ${ }^{13}$ and significance was set at $p=0.05$.

\section{Results}

CLINICAL ASSESSMENT

There was a significant increase in the number of interphalangeal nodes over the 18 months from a mean (SD) for each patient of $3 \cdot 4(4 \cdot 5)$ to $7 \cdot 5(5.4)(p=0.0014)$. The mean (SD) size for each patient increased from $9 \cdot 6(12)$ to $21(17 \cdot 4)$ $(p=0.002)$. The mean (SD) number of tender joints per patient at entry was $7 \cdot 7(6 \cdot 9)$. The mean severity for the tender joints was $9 \cdot 4(8 \cdot 4)$. Neither changed significantly.

\section{MICROFOCAL RADIOGRAPHY}

A detailed description of the macroradiographic features and progression of hand osteoarthritis has been reported elsewhere, ${ }^{10}$ and the principal findings are summarised below.

Subchondral sclerosis At baseline all patients with osteoarthritis had significantly increased subchondral cortical thickness in the right $(p=0.003)$ and left $(p=0.002)$ wrist and hand. By 18 months the subchondral cortical thickness had increased significantly in the patients with osteoarthritis as a group $(p=0.006)$. Three groups were identified on the basis of their radiographic progression: $19(60 \%)$. were considered to have progressed because each patient showed an increase in subchondral sclerosis greater than the coefficient of variation of $4 \%$. Two patients $(6 \%)$ showed no change in subchondral cortical thickness, whereas the remaining 11 patients (34\%) showed a significant decrease $(\mathrm{p}<0.005)$.

Osteophytes were noted at baseline visit in the right and left hands of all patients compared with controls. During the 18 month period osteophytes in both hands increased significantly in both number $(p<0.005)$ and area $(p<0.005)$.

fuxta-articular radiolucencies were seen in the bones of the wrist and finger joints of all patients with osteoarthritis compared with controls. During the study period these radiolucencies showed no significant change in number but increased significantly in size $(\mathrm{p}=0.002)$.

Foint space width Two main groups were identified from the baseline measurements: 18 (56\%) patients with osteoarthritis had a joint space slightly but not significantly wider than the non-arthritic subjects in the right and left wrist and hands. The remaining 14 (44\%) patients had a joint space significantly narrower than the non-arthritic controls. These two groups were further subdivided into four groups (A, B, C, and D) on the basis of radiographic progression: A, 7 (22\%) were normal at entry and remained unchanged; B, 11 (34\%) normal at entry and narrowed; C, 10 (31\%) narrow at entry but did not change; $D, 4$ (13\%) narrow at entry and narrowed further.

\section{BONE SCAN}

All patients had one or more joints which scored one or more on the scan. At entry the mean (SD) number for each patient was 14.5 (5.8) and the mean (SD) intensity score was $34 \cdot 3(19 \cdot 4)$. There was no significant change in these means at 12 months.

Comparison of the distribution of joints with a positive scan in the hands at entry with that at 12 months showed a similar pattern in both hands with the following joints being most commonly affected: thumb metacarpophalangeal joint $(62 \%)$, index distal interphalangeal 
Mean (standard deviation) of the changes in osteophyte number and area during 12 months grouped by change in the bone scan score. The significance of differences was calculated between the group with raised or increasing isotope uptake and the group with decreasing or no radionuclide uptake.

\begin{tabular}{llllll}
\hline Group & $\begin{array}{l}\text { Number of } \\
\text { joints }\end{array}$ & $\begin{array}{l}\text { Change in } \\
\text { number }\end{array}$ & $\begin{array}{l}\text { Combined groups } \\
\text { change in } \\
\text { number }\end{array}$ & $\begin{array}{l}\text { Change in area } \\
\left(\text { mmm }^{2}\right)\end{array}$ & $\begin{array}{l}\text { Combined group } \\
\text { change in } \\
\text { area }\left(\mathrm{mm}^{2}\right)\end{array}$ \\
\hline Decrease in score & 216 & $0.06(0.08)$ & $0.15(0.74)^{*}$ & $0.007(1.63)$ & $0.08(1.32) \dagger$ \\
Unchanged score: 0 & 349 & $0.20(0.67)$ & $0.09(1.25)$ & $0.45(3.59)$ & $0.51(2.91) \dagger$ \\
Unchanged score: $1-4$ & 188 & $0.14(1.01)$ & $0.22(1.03)^{*}$ & $0.62(2.25)$ & \\
Increase in score & 109 & $0.35(1.06)$ & & & \\
\hline
\end{tabular}

*Not significant; $\nmid p<0.005$ (Mann-Whitney U test).

joint (59\%), thumb distal interphalangeal joint (59\%), carpometacarpal joint (59\%), and middle distal interphalangeal joint (59\%). There were slightly fewer joints with a positive scan in the left hand (216 compared with 238). Also, the severity score was lower in the left hand ( 517 compared with 554). The wrist showed considerable scintigraphic activity, with 16 (50\%) patients having a positive scan in the ulnar compartment and $10(31 \%)$ in the radial compartment.

Statistical analysis showed the following significant correlations at the first visit; scan and area of osteophytes $(p=0.003$ for the right hand and $p=0.0013$ for the left hand); scan and joint tenderness $(p=0.0037$ for the right hand and $p=0.0004$ for the left hand); number of radiological osteophytes and nodes $(p=0.0004$ for the right hand and 0.0015 for the left). Correlation between joint tenderness and nodes was highly significant in the left hand $(p=$ 0.0005 ) but just failed to reach significance in the right $(p=0.059)$.

In $13(41 \%)$ patients there was a change in the number of joints with a positive scan between the first and second visit. The table shows the mean change in osteophyte number and area during the one year period grouped by change in bone scan. At joints where there was either a decreased or negative score only a very small increase in osteophyte was detected. At joints where there was either a positive but unchanged or an increased score there was a significant increase in osteophyte size but not in number. No correlation was found between the bone scan and the extent or the change in the dimensions of subchondral sclerosis, joint space width, and juxta-articular radiolucencies.

\section{Discussion}

Osteoarthritis is not a disease in the conventional sense but rather a process of joint disintegration with many causes, characterised by cartilage loss and reactive new bone formation at joint margins with associated variable degrees of synovitis and fibrous thickening of the joint capsule. In the large weightbearing joints, such as the hip and knee, abnormal development and trauma are implicated as causes but the cause of osteoarthritis of the hands is unknown and may be multifactorial with an underlying predisposition and genetic factors having an important role. ${ }^{14}$ Radiologically, osteoarthritis, is common so that it becomes difficult to determine what is the result of disease and what reflects normal aging, particularly when despite radiological change there is no pain or interference with joint function. ${ }^{15}$

Radiographs reflect the morphology of mineralised bone and show the result of repair and destruction. A scan is a study of function and depends on osteoblastic and osteoclastic activity and bone vascularity and reflects the dynamic response of bone to a variety of insults, such as trauma, inflammation, and neoplasia. ${ }^{16}$

A comparison of diagnostic modalities reported that scintigraphy showed more extensive abnormality than either radiography or arthrography in osteoarthritis of the knee. ${ }^{17}$ In their paper comparing bone scanning with conventional radiography Hutton et al studied 33 patients with nodal osteoarthritis of the hands and showed an overall similar pattern of involvement between scanning and radiography but a marked dissimilarity in some patients between the severity on radiography compared with scanning. ${ }^{3}$ Some joints were found to be abnormal on one or other investigation only. They also noted that a joint with a positive scan was likely to show subsequent radiological deterioration, but they were unable to locate the site of deterioration or measure this because of the poor resolution of conventional hand radiographs. ${ }^{4}$

The site of uptake of radiopharmaceuticals in bone has been studied in experimentally induced osteoarthritis in rabbits. In early disease uptake is concentrated in developing osteophytes and, later, in the subchondral bone. In human femoral heads with end stage osteoarthritis, removed at operation after injection of bone seeking isotopes, autoradiography showed uptake primarily in the subchondral bone and in the walls of cysts. ${ }^{18}$

Increased thickness of subchondral bone is a major feature of osteoarthritis and was present in all our patients. It increased radiologically in $19(60 \%)$ but we found no correlation between this feature and the scan, despite the animal models. There may be two stages of sclerosis: an early stage, which may be the response to initiating factors such as subchondral microstress fractures, ${ }^{1920}$ and a later one, which represents the response to altered weight bearing consequent on cartilage loss and disordered alignment. In the hand neither process seems scintigraphically active, but this may be because of the difference in size of the joints-those of the hand being much smaller than the hip in man and the knee in the rabbit-or the degree of loading to which the hand joints are subjected. 
Juxta-articular radiolucencies are not reported to be a characteristic feature of the radiography of osteoarthritis and their exact nature is unclear, but they may be an important early feature. The similarity of their distribution to that of rheumatoid erosions ${ }^{10}$ suggests that they may have an inflammatory origin. We found no correlation between juxta-articular radiolucencies, tenderness, and a positive scan, however, which makes this unlikely, and their exact origin and significance remain obscure despite the fact that they were present in all patients and increased significantly in size.

A cardinal feature of osteoarthritis is loss of articular cartilage. In the early stages disruption of the collagen matrix results in swelling as water content is increased, and this is followed by fissuring and loss of cartilage substance. ${ }^{21}$ In just over half of our patients we found a slightly increased joint space, suggesting that microfocal radiography could detect cartilage swelling in early disease. In the others there was already established thinning. In the subgroups described, in whom progressive cartilage loss was shown on serial radiographs, no correlation was found with the scan, so this key process in osteoarthritis seems to be scintigraphically inert, at least in the wrist and hand.

When the data were analysed according to the presence and growth of osteophytes, highly significant correlations were found with the scan and to a lesser extent with joint tenderness, though this finding must be interpreted with caution as the patients were allowed to take analgesic and anti-inflammatory drugs as required. Osteophyte formation seemed to be a key process in causing clinical symptoms. If the work of Hutton et al is correct osteophytes would also seem to be the marker for subsequent disintegration of the joint architecture as this was predicted by a positive scan and hence, as our study has shown, by the presence of actively remodelling osteophyte. ${ }^{4}$ This is a surprising finding in view of the previous suggestion that osteophytes may be an independent phenomenon related to age. ${ }^{15}$ Possibly, the rate of remodelling of osteophyte is the key: the slower the change in size, the less likely that the joint is seriously compromised. Previously scintigraphy was considered a sensitive but nonspecific tool in osteoarthritis, but this study has shown that it detects very specific pathological processes of clinical significance. With the development of chondroprotective agents more studies are likely to be carried out in patients with osteoarthritis. In a disease with such a long, intermittent, and poorly described natural history, scintigraphic grading of disease activity may prove a useful way of standardising treatment groups. The role of osteophytes in the causation of symptoms in osteoarthritis in large weightbearing joints also merits further study.

This study was supported by the international cartilage project of Ciba Geigy Pharmaceuticals. The authors would like to thank Dr M K Jasani, Mr I Carmichael, and Mrs H Rees for their assistance.

1 Kellgren J H, Lawrence J S. Radiological assessment of osteoarthritis. Ann Rheum Dis 1957; 16: 494-501.

2 Rosenthall L. Osteoarthritis. In: Fogelman I, ed. Bone scanning in clinical practice. Berlin-Heidelberg: Springer 1987: $142-4$.

3 Hutton C W, Higgs E R, Jackson P C, Watt I, Dieppe P A. Tc HMDP bone scanning in generalised nodal osteo arthritis. I. Comparison of the standard radiograph and four hour bone scan image of the hand. Ann Rherm Dis 1986; 45: 617-21.

4 Hutton C W , Higgs E R, Jackson P C, Watt I, Dieppe P A ${ }_{99 m}$ Tc HMDP bone scanning in generalised nodal osteoarthritis. II. The four hour bone scan image predicts arthritis. If. The four hour bone scan image predic
radiographic changes. Ann Rheum Dis 1986; 45: 622-6.

5 Buckland-Wright $J C$. A new high definition microfocal $x$-ray unit. Br $\mathcal{Y}$ Radiol 1989; 62: 201-8.

6 Buckland-Wright J C, Bradshaw C R. Clinical applications of high definition microfocal radiography. $\mathrm{Br} \mathcal{J}$ Radiol 1989 ; 62: 209-17.

7 Buckland-Wright J C, Carmichael I, Walker SR. Quantitative microfocal radiography accurately detects joint changes in rheumatoid arthritis. Ann Rhewn Dis 1986; 45: 379-83.

8 Buckland-Wright J C. X-ray assessment of activity in rheumatoid disease. $B r$ F R herumatol 1983; 22: 3-10.

9 Doyle D V, Dieppe P A, Scott J, Huskisson E C. An articular index for assessment of osteoarthritis. Ann R heum Dis 1981; index for $40: 75$.

10 Buckland-Wright J C, Macfarlane D G, Lynch J A, Clark B. Quantitative microfocal radiographic assessment of progression in osteoarthritis of the hand. Arthritis Rheum 1990, 33: 57-65.

11 Altman R D, Fries J F, Bloch D A, et al. Radiographic assessment of progression in osteoarthritis. Arthritis Rhew 1987; 30: 1214-25.

12 Buckland-Wright J C, Walker S R. Incidence and size of erosions in the wrist and hand of theumatoid patients: a quantitative microfocal radiographic study. Ann $R$ heum $D$ is quantitative mict

13 Norusis M J. SPSS/PC+ for the IBM PC/XT/AT. Chicago: SPSS Inc, 1986

14 Kellgren J H, Lawrence J S, Bier F. Genetic factors in generalised osteoarthritis. Ann Rheum Dis 1963; 22 : $237-55$

15 Wood P H N, Badley E M. Epidemiology of individual rheumatic complaints. In: Scott J T, ed. Copeman's textbook of the rherumatic diseases. 6th ed. Edinburgh: Churchill Livingstone, 1989: 59-142.

16 Merrick M V. Bone scanning. Br I Radiol 1975; 48: 327-51.

17 Thomas R H, Resnick D, Alazraki N P, Daniel D, Greenfield R. Compartmental evaluation of osteoarthritis of the knee. Radiology 1975; 116: 585-94.

18 Christensen S B. Osteoarthritis: changes of bone, cartilage and synovial membrane in relation to bone scintigraphy. and synovial membrane in relation to

19 Radin E L, Paul I L, Rose R M. Role of mechanical factors in the pathogenesis of primary osteoarthritis. Lancet 1972; i 519-22.

20 Duncan $\dot{H}$, Riddle J M, Jundt J W, Pritchard W. Osteoarthritis and the subchondral plate. In: Verbruggen $G$, Veys E M, eds. Degenerative joints. Vol 2. Amsterdam Excerpta Medica, 1985: 181-91.

21 McLevitt C A, Muir H. Biochemical changes in the cartilage of the knee in experimental and natural osteoarthritis in the dog. $\mathcal{F}$ Bone foint Surg [Br] 1976; 58: 94-8. 